\title{
RGB optimization in a Top-emission Organic Light-emitting Device
}

\author{
Yung-Ting Chang, Hung-Chi Chen, Jiun-Haw Lee*, Yean-Woei Kiang, and C. C. Yang \\ Graduate Institute of Electro-Optical Engineering, Graduate Institute of Communication \\ Engineering, and Department of Electrical Engineering, National Taiwan University, No. 1, Sec. 4, \\ Roosevelt Road, Taipei, Taiwan, R.O.C.
}

\begin{abstract}
In this paper, we demonstrate simulation results of top-emission organic light-emitting devices (TOLEDs) with red, green and blue (RGB) colors. We take the RGB spectral peaks at 460, 520, and $600 \mathrm{~nm}$ with full width at half maximum of $100 \mathrm{~nm}$. Device structures are thick silver (Ag) anode /hole-transport layer $60 \mathrm{~nm} /$ emitting layer (EML) $/ \mathrm{semi}$ transparent Ag cathode $20 \mathrm{~nm} /$ dielectric layer. The dielectric material capped upon the cathode is zinc selenium with a refractive index of 2.6 for providing high output intensity and narrow FWHM. When monitoring the peak wavelengths of RGB device and varying the EML and dielectric thicknesses, we found the optimized value of the EML are 71, 47 and $31 \mathrm{~nm}$ for the red, green and blue devices, respectively. The optimized dielectric thicknesses are 80,70 and $50 \mathrm{~nm}$ with periods of 117, 98 and $90 \mathrm{~nm}$, respectively, for the RGB devices. Due to the limitations of the experiments, the EML thicknesses can be different and the dielectric thickness must be the same of the RGB devices. For optimizing the BGB devices simultaneously, the thickness of dielectric layer of the OLED is $667 \mathrm{~nm}$. The RGB peak intensities are $0.96,0.99$ and 0.83 , normalized to their optimized value. Typically, in a TOLED, green device exhibits higher efficiency than red and blue ones. That means the intensity of the green TOLED can be lower. When the dielectric layer thickness is $314 \mathrm{~nm}$, the normalized RGB peak intensities are 0.99, 0.26 and 0.97 .
\end{abstract}

Keywords: organic light-emitting device, simulation, electromagnetic theory

\section{INTRODUCTION}

Organic light-emitting devices (OLEDs) have attracted much attention due to the advantages low driving voltage, self emissive, wide-view-angle, flexible-substrate-capability, and potentially low cost [1], [2]. Top-emission OLED (TOLED) is one of the most promising technologies for achieving high aperture ratio active matrix OLED [3]. Typically, such a device consists of a reflective anode, a p-type and n-type organic layers, and a semi-transparent metal cathode. Electro-luminescence (EL) is generated from the organic layers and coupled out of the device from the semitransparent metal side. Due to the high reflective anode and semitransparent metal cathode, there is strong microcavity effect in a TOLED. To improve the optical intensity and color purity, usually, TOLED is capped with a dielectric layer [4]. When varying the thickness of the dielectric layer, optical intensity from the OLED has an oscillatory behavior due to the constructive or destructive interferences. For a full-color OLED, three primary colors (red, green, and blue; RGB) must be optimized simultaneously with a common dielectric layer to achieve the maximized intensity. Hence, the thickness of this layer is critical to the three primary colors.

In this research, we develop a rigorous and efficient numerical method to simulate the radiation characteristics of a TOLED with RGB colors. In particular, we intend to establish a design rules for the RGB TOLEDs with some common structures. By adjusting the layer thicknesses of the organic layers, semi-transparent metal, and dielectric layers, we can find the optimized optical characteristics for the RGB pixels, respectively, for a guideline in device design. Besides, during device fabrications, there are some common layers for RGB sub-pixels to reduce the fabrication cost. This factor is also included in our optical model. In this paper, the theoretical formulations are given in section 2. Numerical results and the related discussions are presented in section 3. Finally, a summary is drawn in section 4.

*jhlee@cc.ee.ntu.edu.tw; phone 8862 2363-5251 ext. 540; fax 8862 2367-7467

Organic Light Emitting Materials and Devices X, edited by Zakya H. Kafafi, Franky So, Proc. of SPIE Vol. 6333, 63331C, (2006) · 0277-786X/06/\$15 - doi: 10.1117/12.678979 


\section{THEORY}

The TOLED device consists of multilayer structure as shown in Fig.1. The devices are fabricated on the glass substrate with an opaque anode Ag. The organic multilayer structure consists of the hole-transporting layer (HTL), the emitting layer (EML), and the electron-transporting layer (ETL). The EL is observed through a semitransparent metal cathode consists of $20 \mathrm{~nm} \mathrm{Ag}$. Finally, a dielectric layer, such as $\mathrm{ZnSe}$, was added to enhance the output coupling efficiency. For RGB subpixels, the only difference comes from the organic materials and thicknesses of the EML. On the other hand, the Ag anode, HTL, ETL, and dielectric layers for RGB subpixels are usually in common for simplifying the fabrication process. For calculating the radiation from dipole sources embedded in the emission layer (EML), we first introduce the electric Hertzian vector potential due to a vertical or horizontal dipole embedded in the EML [5]. Then, with $e^{j o t}$ timeharmonic dependence, the electric and magnetic fields can be derived from the Hertzain vector potential.

$E=k_{0}{ }^{2} n^{2} \Pi+\nabla \nabla \cdot \Pi$

$H=-j \omega \varepsilon_{0} n^{2} \nabla \times \Pi$

where $\mathrm{n}$ is the complex refractive index and $k_{0}=\omega \sqrt{\mu_{0} \varepsilon_{0}}=2 \pi / \lambda$ is the wave number or propagation constant in vacuum. The wave equations for $\Pi$ can be expressed as

$$
\begin{aligned}
& \left(\nabla^{2}+n_{i}^{2} k_{0}^{2}\right) \Pi=0 \quad, \quad \text { in } \quad i^{\text {th }} \quad \text { (non-emission) layer } \\
& \left(\nabla^{2}+n_{e}{ }^{2} k_{0}^{2}\right) \Pi=\frac{-p \delta\left(r-r^{\prime}\right)}{\varepsilon_{0} n_{e}^{2}}, \text { in EML }
\end{aligned}
$$

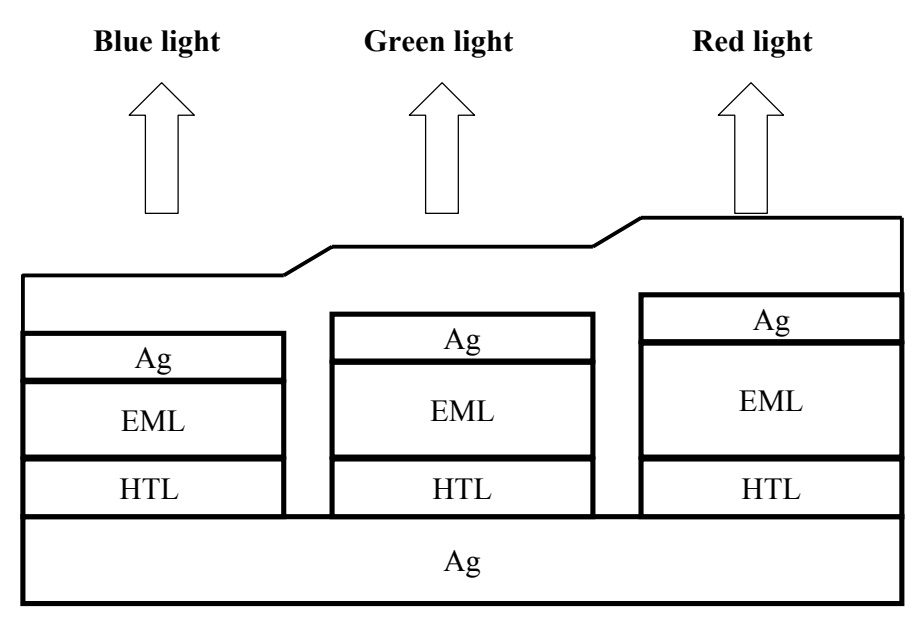

Fig. 1 Device structure of RGB-TOLEDs.

where $\mathrm{p}$ denotes a dipole source which is located at $\mathrm{r}=\mathrm{r}$ and $\mathrm{n}_{\mathrm{e}}$ is the wavelength-dependent complex refractive index for the EML. We employ the property of axial symmetry and take the Fourier-Bessel transform to obtain an integral expression for the Hertzian vector potential in each layer of OLED. By using the boundary conditions that the tangential electric and magnetic fields are continuous across the interface between layers, we can determine the unknown coefficients in the integral expression of Hertzian potential from which all the electromagnetic field components can 
then be calculated accordingly. To calculate the radiation field in the far region, the saddle-point method is usually used to perform the integration over the radial wavenumber [6]. For a dipole source operating at a single frequency, strong interference of the coherent fields reflected from interfaces between layers results in a rapidly oscillatory radiation pattern. In fact, every real light source should have a finite bandwidth and the interference will be smoothened. Therefore, we assume that the dipole source is of a finite bandwidth (say, $0.5 \mathrm{~nm}$ ), and the resultant radiation field is obtained by coherently averaging the fields over that range of wavelengths.

Based on the above theory, we establish a simulation program. The dipole was decomposed into a vertical dipole component and horizontal dipole component. First we calculate the $\mathrm{x}, \mathrm{y}$ and $\mathrm{z}$ components of the radiated field from horizontal and vertical dipole embedded in the emission layer, respectively. Then we add up coherently the radiated fields due to both the vertical dipole and horizontal dipole component of a dipole oriented in the random direction. We can compute the resulting the resulting intensity due to a large number randomly oriented dipole sources by incoherently adding the radiated intensity due to each dipole and then taking the average. The input parameters of this program are the thicknesses and refractive index (the refractive index can be complex) of each layer and the PL spectrum of the emitting material. The outputs of this simulation program include the radiant intensity pattern due to randomly oriented dipoles, and the electroluminescence emission patterns for a given photoluminescence. The output data are the radiant intensity pattern due to randomly oriented dipoles, the electroluminescence emission patterns for a given photoluminescence, polarization CIE coordinate and optical intensity of any view angle.

\section{RESULTS AND DISCUSSIONS}

Fig. 2 (a), (b), and (c) show the optical intensity variation of the RGB subpixels with dielectric layer thicknesses for different EML thicknesses, respectively. The Ag anode is opaque and the refractive index of the dielectric layer is 2.6. The thicknesses of the HTL and the semi-transparent Ag are 60 and $20 \mathrm{~nm}$, respectively, which are optimized following the design rules as shown in ref. 5. The monitoring wavelengths of the red, green, and blue colors are fixed at 460, 520, and $600 \mathrm{~nm}$, respectively. We can see that, for Fig. 2(a), (b) and (c), the optical intensity has an oscillatory behavior when varying the dielectric thickness due to the interference effect. Such a behavior can be explained by using the planar microcavity effects [7]. Besides, when varying the EML thickness, for example in blue OLED of Fig. 2 (a), the optical intensity increases then decreases and has a maximized optical intensity at around $30 \mathrm{~nm}$. After optimizing the EML thickness and the dielectric layer, we obtain the maximum optical intensity at 460, 520 and $600 \mathrm{~nm}$ at the EML thickness of $31 \mathrm{~nm}$ and dielectric layer thickness of $50 \mathrm{~nm}$ for blue device, the EML thickness of $47 \mathrm{~nm}$ and dielectric layer thickness of $70 \mathrm{~nm}$ for green device, and the EML thickness of $71 \mathrm{~nm}$ and dielectric layer thickness of $80 \mathrm{~nm}$ for red device, respectively. The oscillatory period of the dielectric layer is 90,98 and $117 \mathrm{~nm}$, respectively, for blue, green and blue OLEDs. During the RGB optimization, the EML thickness should not exceed $100 \mathrm{~nm}$ for better electrical performances. On the other hand, the thickness of the dielectric layer can be increased for adjusting the out-coupling intensity.

Proc. of SPIE Vol. 6333 63331C-3 


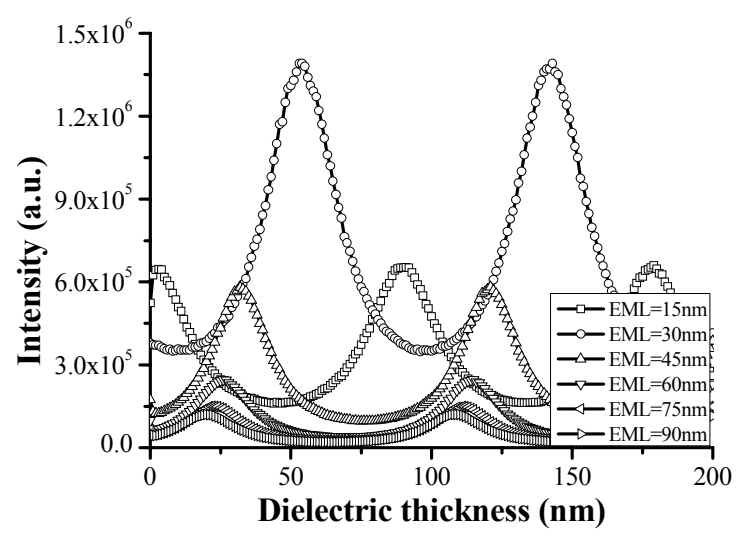

(a)

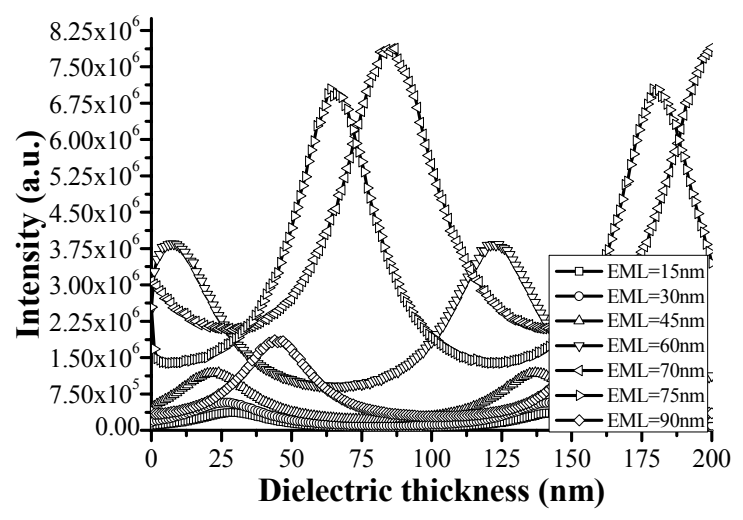

(c)

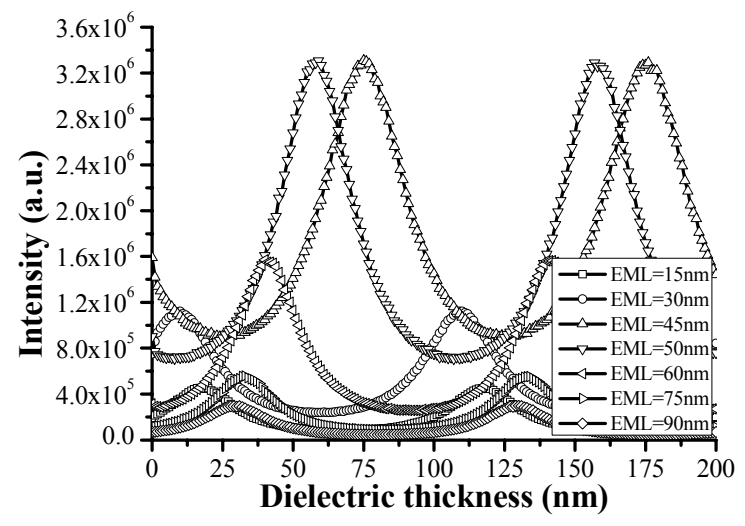

(b)

Fig. 2 Intensity (viewing angle $=0^{\circ}$ ) as a function of different EML thicknesses when using different dielectric layer thicknesses. OLED structure: Ag/ 60nm HTL/ Z nm EML / 20nm Ag/ X nm dielectric layer (refractive index =2.6). (a) blue device with the wavelength of $460 \mathrm{~nm}$, (b) green device with the wavelength of $520 \mathrm{~nm}$, and (c) red device with the wavelength of $600 \mathrm{~nm}$.

After determining the layer thicknesses of the RGB subpixels, we then simulate their respective spectra with different dielectric thicknesses as shown in Fig. 3. We can see that when the dielectric layer thicknesses are optimized, the optical intensities are highest and the FWHM are smallest for RGB subpixels, in Fig. (a), (b), and (c). 


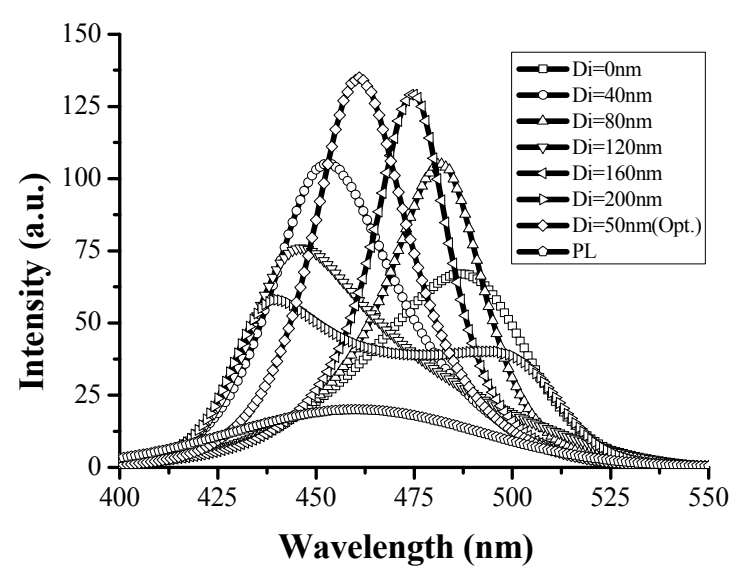

(a)

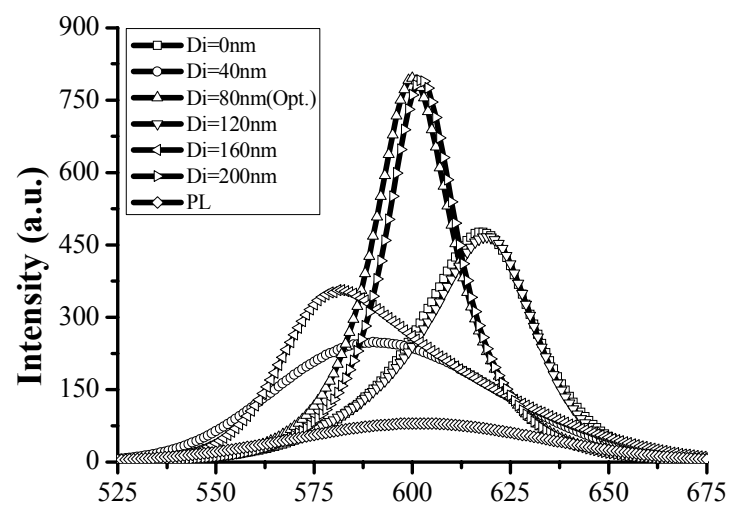

Wavelength (nm)

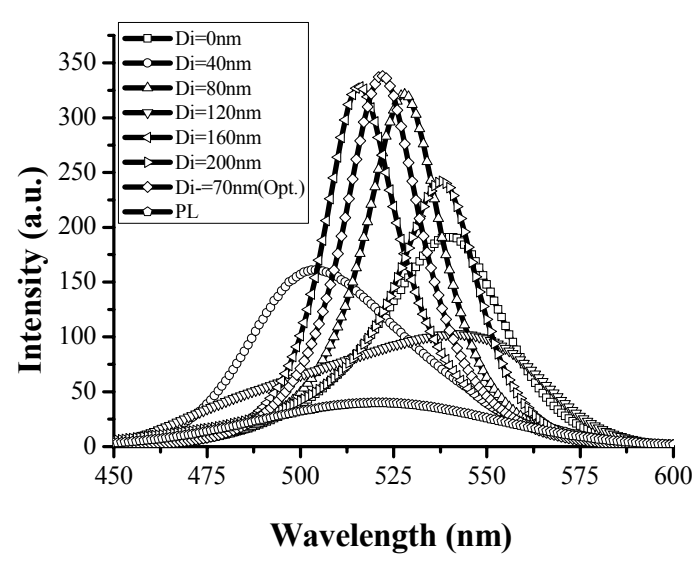

(b)

(c)

Fig. 3 Spectra (at viewing angle $=0^{\circ}$ ) with different dielectric layer thicknesses. OLED structure: Ag/ 60nm HTL/ Z nm EML / 20nm Ag/X nm dielectric layer (refractive index=2.6). (a) Blue device $Z=31 \mathrm{~nm}$. (b) Green device $Z=47 \mathrm{~nm}$. (c) Red device $Z=88 \mathrm{~nm}$.

However, as discussed before, a common dielectric is needed during fabrication. For optimizing the RGB devices simultaneously, we simulate the optical intensity with different dielectric layer for RGB pixels as shown in Fig. 4. The EML thicknesses of RGB are 88, 47, and $31 \mathrm{~nm}$, respectively. We can see that at $667 \mathrm{~nm}$, the RGB pixels are nearly optimized with their respective intensity of $0.83,0.99$ and 0.96 , normalized to their optimized value. However, such a layer may be too thick in fabrication. Typically, in a TOLED, green device exhibits higher efficiency than red and blue ones. That means the intensity of the green TOLED can be lower. When the dielectric layer thickness is $314 \mathrm{~nm}$, the normalized RGB peak intensities are $0.99,0.26$ and 0.97 . 


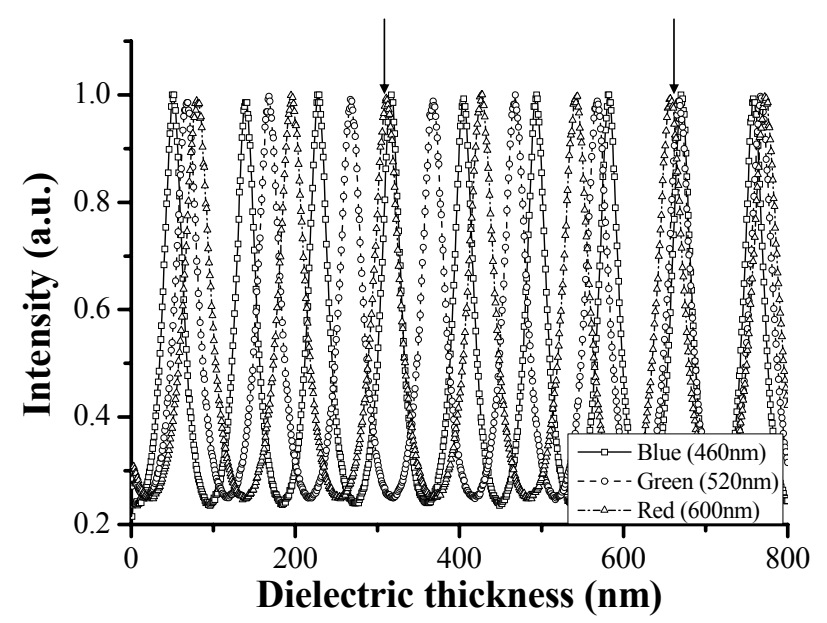

Fig. 4 Intensity (viewing angle $=0^{\circ}$ ) as a function of dielectric layer thicknesses for RGB subpixels.

\section{SUMMARY}

In this paper, we demonstrate a RGB subpixel optimization of OLEDs based on the common HTL, cathode, and dielectric structure. Organic materials and layer thicknesses of the EML of RGB subpixels are varied to achieve the maximum optical intensity. Besides, since the dielectric layer for the RGB subpixels are in common, an optimized thickness is needed. For maximizing the RGB simultaneously, the dielectric layer thickness is $667 \mathrm{~nm}$. Since green emission for an OLED is typically highest, the relative intensity can be lower compared to red and blue OLEDs. Hence, a dielectric layer with thinner thickness of $314 \mathrm{~nm}$ is chosen in our optimization OLEDs.

\section{ACKNOWLEDGMENTS}

This work was financially supported by Vitex Systems Incorporation.

\section{REFERENCES}

1. C. W. Tang and S. A. Vanslyke, Appl. Phys. Lett. 51, 913 (1987).

2. S. A. Van Slyke, C. H. Chen, and C. W. Tang, Appl. Phys. Lett., 69, 2160 (1996).

3. H. Riel, S. Karg, T. Beierlein, W. Rieß, and K. Neyts, J. Appl. Phys 945290 (2003)

4. H. Riel, S. Karg, T. Beierlein, B. Ruhstaller, and W. Rieß, Appl. Phys. Lett. 82466 (2003)

5. J. H. Lee, K. Y. Chen, C. C. Shiau, C. C. Yang, and Yean-Woei Kiang, IEEE/OSA J. of Display Technology 2130 (2006)

6. K. B. Kahen, Appl. Phys. Lett. 781649 (2001)

7. A. Ishimaru, "Electromagnetic Wave Propagation, Radiation, and Scattering" London: Prentice-Hall International (1991) 